\title{
Seismic Wave Propagation Through a Low-Velocity Nuclear Rubble Zone
}

Shawn Larsen

David Harris

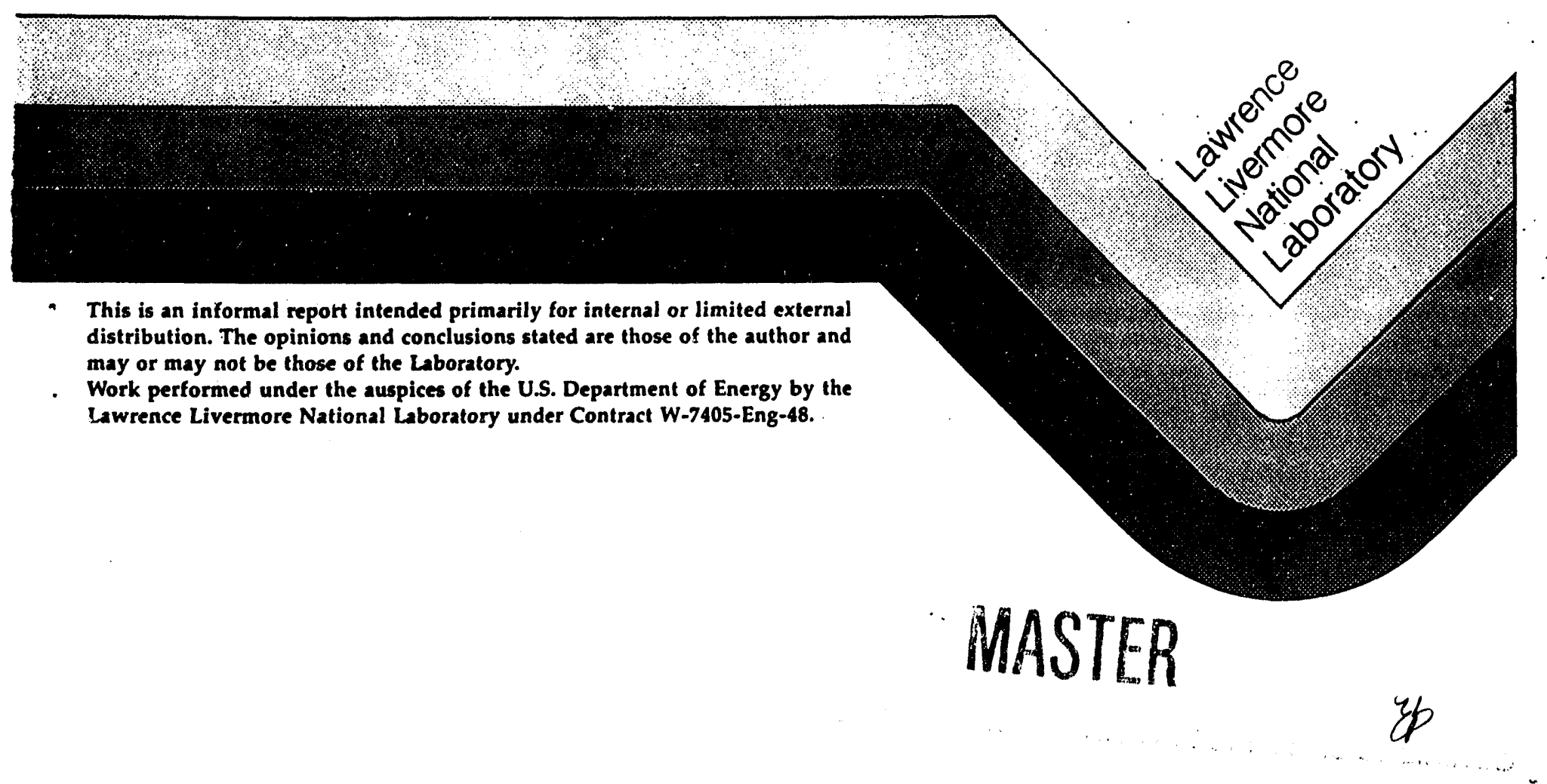




\section{DISCLAIMER}

This document was prepared as an account of work sponsored by an agency of the United States Government. Neither the United States Government nor the University of California sor anj of their employees, makes any warranty, express or implied, or assumes any legal liability or responsibility for the accuracy, completeness, or usefulness of any information, apparatus, product, or process disclosed, or represents that its use would not infringe privately owned rights. Reference herein to any specific commerdal products, process, or service by trade name, trademark, manufacturer, or otherwise, does not mecesarily constitute or impiy its endorsement, recommendation, or favoring by the United States Goverwment or the Ualversity of Calffornin. The views and opinions of authors expressed herein do not necessarily state or renect those of the United States Government or the University of California, and shall not be used for advertising or product endorsement purposes.

This report has been reproduced directly from the best available copy.

Avalable to DOE and DOE contractors from the Orfice of Scientific and Technical Information P.O. Box 62, Oak Ridge, TN 37831

Prices available from (615) 576-8401, FTS 626-8401

Available to the public from the

National Technical Information Service

US. Department of Commerce 5285 Port Royal Rd,

Springfield, VA 22161 
Seismic Wave Propagation Through a Low-Velocity Nuclear Rubble Zone

\author{
Shawn Larsen and David Harris \\ Lawrence Livermore National Laboratory \\ Livermore, CA 94551
}

\begin{abstract}
A 2-dimensional finite-difference code has been developed as part of the Hydroacoustic Nuclear Proliferation Monotoring Project (ST639). This code is used to model seismic and acoustic wave propagation in the Earth's crust and oceanic water column. As a demonstration, we investigate the propagation of seismic energy through a low-velocity region corresponding to the rubble zone created by an underground nuclear explosion. The geometry of the simulated source and surface recording instruments is representative of a seismic refraction survey. The purpose of this numerical simulation is to determine if perturbations in the seismic wave field due to the low-velocity region (nuclear cavity) can be detected at the surface. Wave energy passing through the cavity is noticeably delayed, and is easily observed on crosssections of the $\mathbf{P}$ and $\mathbf{S}$ wave fields. Synthetic seismograms recorded at the surface demonstrate an amplitude reduction and phase shift for those stations corresponding to ray-paths through the cavity. A significant fraction of energy is reflected off the lowvelocity zone, and propagates as backward traveling waves.
\end{abstract}

\title{
INTRODUCTION
}

Nuclear non-proliferation has become one of the most important security issues in the world today. One focus of DOE research is the development and evaluation of techniques to detect clandestine nuclear tests. A potential method is the use of hydroacoustic monitoring devices in the ocean to discriminate suspected events on land. As part of the Hydroacoustic Nuclear Proliferation Monotoring Project (ST639), we developed a finite-difference code to numerically simulate seismic and acoustic wave propagation. Our main objective is to investigate wave propagation from the continental crust (and upper mantle) out into the oceanic water column. In this report, however, we demonstrate the finite-difference code by focusing on a specific application involving on-site inspection to detect underground nuclear events by seismic techniques. We find that seismic methods are potentially advantageous as a near-source discriminant.

An underground nuclear explosion vaporizes and compresses the surrounding geologic material leaving behind an sub-surface cavity. This cavity is structurally unstable and tends to collapse forming an anomalous region known as a rubble zone. Seismic velocities in the rubble zone are considerably less than the surrounding material. Seismic techniques can be used as a mechanism for detecting this low-velocity region, and hence suspected nuclear events.

In this study we investigate the use of seismic refraction methods to detect the cavity created by an underground nuclear event. We simulate a refraction experiment by utilizing finite-difference techniques, specifically investigating the effects of simulated wave propagation through a low-velocity zone. We find a noticeable delay for seismic 
energy passing through this region. In addition, a significant fraction of energy reflects off the rubble zone and propagates backward. These perturbations in the seismic field are illustrated by vertical cross-sections of the wave field and by synthetic seismograms positioned along the surface.

\section{FINITE DIFFERENCING}

In seismic applications, finite-difference methods are typically used for numerical solutions to the full wave equation [Kelly et al., 1976; Charrette, 1992]. The method is general and flexible, and may be applied to an arbitrary inhomogeneous body of any shape. Because of its simplicity, implementation is made with minimal analytical effort. Finite-difference numerical simulation methods are frequently employed by oil-industry geophysicists to model seismic reflection data [e.g., Claerbout, 1985], and by earthquake seismologists studying the source, path, and site effects associated with seismic events [e.g., Graves and Clayton, 1992; Frankel and Vidale, 1992].

In this study a 4th-order finite-difference scheme is used to model seismic wave propagation in a fully elastic 2-D inhomogeneous medium [e.g., Levander, 1988]. This algorithm is based on a staggered-grid implementation of the elastodynamic formulation of the full wave equation [Madariaga, 1976; Virieux, 1986]. First-order absorbing boundary conditions using paraxial extrapolators are applied to the side and bottom grid interfaces [Clayton and Engquist, 1977, 1980]. Mode conversions at the free-surface are modeled correctly using stress-free boundary conditions [e.g., Vidale and Clayton, 1986]. An explosive source with a time history defined by a Ricker wavelet is used as the driving term. More information about the finite-difference techniques are given in the appendix.

We solve for the radial $\left(V_{x}\right)$ and vertical $\left(V_{2}\right)$ velocities of particle motion, along with the normal $\left(\tau_{x x}, \tau_{z z}\right)$ and shear $\left(\tau_{x z}\right)$ components of the stress tensor. However, it is instructive to write the solution as the time-derivative of the dilatation and shear rotation. The dilatation (time-derivative) is given by

$$
P=\frac{\partial V_{x}}{\partial x}+\frac{\partial V_{z}}{\partial z}
$$

and the shear rotation (time-derivative) is given by

$$
S=\frac{1}{2}\left(\frac{\partial V_{x}}{\partial z}-\frac{\partial V_{z}}{\partial x}\right)
$$

The dilatation is within a srale factor of the $P$ potential and the shear rotation is within a scale factor of the S potential [e.g., Aki and Richards, 1980], so for simplicity we refer to Equations $1-a$ and $1-b$ as the $P$ and $S$ potentials. The decomposition of the elastic solution into these two independent quantities greatly simplifies the visual representation of the seismic wave characteristics. For example, the $\mathrm{P}$ and $\mathrm{S}$ potentials can be displayed as two different colors so that reflections, refractions, and mode conversions at the free surface and medium boundaries are easily identified. This greatly simplifies analysis of the numerical simulations.

\section{GEOMETRY AND CRUSTAL MODEL}

We model a crustal cross-section $6 \mathrm{~km}$ long and $1 \mathrm{~km}$ deep (Figure 1). The P-wave 
velocity of the medium $\left(V_{p}\right)$ is $5.0 \mathrm{~km} / \mathrm{sec}$ along the surface, with a vertical gradient of $2.0 \mathrm{~km} / \mathrm{sec} / \mathrm{km}(7.0 \mathrm{~km} / \mathrm{sec}$ at $1.0 \mathrm{~km}$ depth). The material properties are that of a Poisson solid, so that the $S$-wave velocity is given by $V_{2}=V_{p} / \sqrt{3}$. The medium is laterally homogeneous except for the inclusion of a low-velocity zone in the middle of the cross-section; this zone represents the nuclear cavity. The top and bottom of the cavity are at depths of $180 \mathrm{~m}$ and $448 \mathrm{~m}$, respectively; the width is $80 \mathrm{~m}$. The P wave velocity in this low-velocity zone is $2.0 \mathrm{~km} / \mathrm{sec}$ and it is characterized as a Poisson solid.

A compressional (P-wave) source is located $4 \mathrm{~m}$ below the surface. The time-history of the source is defined by a Ricker wavelet, and is given by

$$
f(t)=-2 \alpha\left(t-t_{0}\right) \exp ^{-\alpha\left(t-t_{0}\right)^{2}}
$$

where $t_{0}$ is the half-width of pulse duration and alpha is a parameter that controls the frequency of the source. For our model $\alpha=20,000, t_{0}=0.025$, and the source initiates at time $t=0$. The maximum frequency for this source is about $100 \mathrm{~Hz}$.

Our finite-difference grid extends for 1500 nodes in the horizontal direction and 250 nodes in the vertical direction (375,000 nodes total). The separation between nodes is $4 \mathrm{~m}$ (in both directions). At a frequency of $100 \mathrm{~Hz}$ and a compressional velocity of 5 $\mathrm{km} / \mathrm{sec}$, the wavelength is $50 \mathrm{~m}$. Thus, our grid has a sampling rate of 10-15 nodes per wavelength. This is sufficient to avoid dispersive effects caused by the finitedifference approximation to the wave equation [e.g., Chin et al., 1984; Kneib and Kerner, 1993; Hedstrom, 1993]. The maximum time-step is constrained by the Courant condition; for our model we use a time-step of $0.00030 \mathrm{~s}$. The simulation is carried out to 3333 time-steps, or a temporal duration of $1 \mathrm{~s}$.

A $5 \mathrm{~km}$ long array of seismometers is positioned along the surface, each sensor separated by $0.2 \mathrm{~km}$. These instruments record the vertical and radial components of the wave field, although the synthetics shown are the $P$ and $S$ potentials. Figure 1 shows the high-frequency ray-paths from the source to each receiver. Several rays pass through the low-velocity zone.

\section{RESULTS}

We ran two simulations: one with and one without the low-velocity rubble zone. Wave propagation through the rubble zone is illustrated in Figure 2, which shows "snapshots" of the progressively expanding wave field at times 0.15 (top), $0.3,0.45$, and 0.6 (bottom) seconds. Red indicates the $P$ potential and green illustrates the $S$ potential (or more precisely, the colors represent the time derivatives of the $P$ and $S$ potentials). A bluish tint indicates negative amplitude. Prominent features in the wave pattern include the direct $P$ arrival $(P)$, the P-to-S conversion along the free-surface (PS), and the Rayleigh wave (R). Due to the shallow depth of the source, a direct shear wave $(S)$ is created by the interaction of the source with the free-surface. Nonabsorbed (artificial) reflections off the bottom and side grid boundaries are noticeable during the later times.

The effect of seismic wave propagation through the low-velocity zone is clearly illustrated in Figure 2. There is a noticeable delay in the direct $P$ pulse that penetrates the rubble zone (Pd). In addition, a significant fraction of energy is reflected back from the nuclear cavity (Pr). Similar propagation characteristics are observed for $S$ 
waves.

Synthetic seismograms from the receiver array along the surface are shown in Figure 3. The seismograms are those corresponding to the $\mathbf{P}$ potential. The first arrival is distinct and uniform for increasing receiver distances up to $3.2 \mathrm{~km}$. At a source distance of $3.4 \mathrm{~km}$, however, the amplitude drops and the initial seismic pulse is delayed. At this distance the synthetic is measuring the ray-path that propagates through the top of the low-velocity zone (Figure 1). This characteristic delay and amplitude reduction is observed for receiver distances up to $4.6 \mathrm{~km}$, where the pulse regains its original shape. It may be possible to use this anomalous feature to detect a sub-surface rubble zone.

The large clipped signal trailing the initial $\mathbf{P}$ arrival corresponds to the surface wave. In a more realistic model (or the real Earth), these arrivals would be smaller due to . near surface attenuation. There is no attenuation in our model.

\section{DISCUSSION}

Figures 2 and 3 demonstrate a measurable perturbation in the seismic signal for energy passing through a low-velocity zone. This is observed as a signal delay and an amplitude reduction. The delay is caused by the longer transmission time through the rubble zone, and the amplitude decrease results from energy being scattered in different directions as the seismic pulse hits the cavity. Notice the high amplitude backward propagating reflection off the void in Figure 2.

To further illustrate the effects of the low-velocity zone, we plot differential seismograms in Figure 4 ( $P$ potential). This figure shows the difference between solutions with and without the void, with the amplitudes the same as in Figure 3. Transmission through the void is indicated by the large differenced signal between 0.5 and 0.7 seconds for receiver distances of 3.4 to $4.6 \mathrm{~km}$. The backward propagating reflection from the low-velocity zone is seen as the arrivals between 0.5 and 0.8 seconds for the near source differenced synthetics $(1.8-2.6 \mathrm{~km})$. Other reflections and refractions from the low-velocity zone arrive at later times for all synthetics.

The amplitude of the perturbed signal (the difference between the two solutions) is comparable to the original signal. This suggests that the low-velocity zone can be detected by seismic methods. This is most true for the first arrival, since later arrivals will be mixed with other signals. There is also a recognizable effect due to the backward propagating reflection from the low-velocity zone.

The real Earth is more inhomogeneous than our simple model. Crustal inhomogeneities will cause perturbations in the seismic signal, in addition to those from the nuclear cavity. This will inhibit the detection of a low-velocity rubble zone (Figures 5, 6, 7, and 8). We have simulated inhomogeneities by applying a Gaussian noise function to the medium parameters. This was accomplished by first randomly perturbing the $\mathrm{P}$ wave velocity at each grid point (following a Gaussian distribution), with no spatial correlation between points. These perturbations were then spatially smoothed by summing all grid points within 7 points about each grid node; this smoothing process was repeated 12 times so that the spatial velocity variations would assume a Gaussian spatial correlation. The grid velocities were then adjusted by a scale factor, so that the 2-sigma deviation would be a specified percentage of the 
original velocities. That is, $10 \%$ velocity "noise" means that $95 \%$ of the grid velocities are within $10 \%$ of the original velocity structure. The $S$ wave velocity was computed so that the P-to-S ratio would be the same as the unperturbed velocity model. The medium density did not change.

Synthetic seismograms for simulations with $5 \%, 10 \%$, and $20 \%$ velocity noise are shown in Figures 5, 6 and 8, respectively. The wave pattern for the $10 \%$ model is shown in Figure 8. In Figure 5, both an amplitude reduction and signal delay are clearly observed for ray paths penetrating the low-velocity region. The $5 \%$ velocity perturbation doesn't have a significant effect on the waveforms. An amplitude reduction is also observed in the $10 \%$ noise model of Figure 6 . In fact, the reduction is even greater than the unperturbed model (Figure 3), but this is due mostly to the particular velocity structure used for the perturbation. The wave fields for the $10 \%$ model are indicated in Figure 7. Although the seismic delay through the void (Pd) and the backwards propagating reflection (Pr) are noticeable, it is clear that the wave fields are beginning to lose their coherency due to the simulated inhomogeneity. Figure 8 shows seismic waveforms for a model with $20 \%$ velocity noise. In this case, the effect of the rubble zone is not discernible. We conclude that the velocity structure needs to be known to about $10 \%$ in order to detect the low-velocity region.

Another factor is that the real Earth is 3 dimensional. Thus, the expected signals will be less pronounced since seismic energy will "wrap around" the low-velocity zone. In addition, it would be more difficult to design an effective seismic refraction experiment in 3 dimensions, since a priori parameters such as the cavity's approximate location would be needed. The largest effect of the low-velocity region is from the direct first arrival passing through the zone. If the cavity is not co-linear with the source and receiver array, then the refraction effects through the rubble zone would not be as pronounced. However, reflected energy from the low-velocity region would remain significant.

\section{ACKNOWLEDGEMENTS}

We thank Gerald Hedstrom, Robert Clayton, and Robert Graves for several discussions and comments about finite-difference methods. This work was performed under the auspices of the Department of Energy by the Lawrence Livermore National Laboratory under contract W-7405-ENG-48. 


\section{APPENDIX}

\section{General Description:}

A 4th-order finite-difference scheme is used to model seismic wave propagation in a fully elastic 2-D inhomogeneous medium. This algorithm is based on a staggered-grid implementation of the elastodynamic formulation of the full wave equation. The algorithm is staggered in both space and time. Low order absorbing boundary conditions using paraxial extrapolators are applied to the side and bottom grid interfaces. Mode conversions at the free-surface are handled correctly using stress-free boundary conditions. A compressional ( $P$ wave) source is used to drive the simulation, with a time history defined by a Ricker wavelet.

\section{Equations:}

We wish to simulate the propagation of seismic waves in a 2-dimensional inhomogeneous elastic medium. The $x$ axis is in the horizontal direction while the $z$ axis is in the vertical direction. The elastic wave equation can be represented in terms of constitutive laws and the equations of motion [e.g., Madariaga, 1976]. The 2-D equations of motion are given by

$$
\rho \frac{\partial V_{x}}{\partial t}=\frac{\partial \tau_{x x}}{\partial x}+\frac{\partial \tau_{x z}}{\partial z}+f_{x}
$$

and

$$
\rho \frac{\partial V_{z}}{\partial t}=\frac{\partial \tau_{x z}}{\partial x}+\frac{\partial \tau_{z z}}{\partial z}+f_{z}
$$

and the constitutive laws are given by

$$
\begin{gathered}
\frac{\partial \tau_{x x}}{\partial t}=(\lambda+2 \mu) \frac{\partial V_{x}}{\partial x}+\lambda \frac{\partial V_{z}}{\partial z} \\
\frac{\partial \tau_{x z}}{\partial t}=\mu \frac{\partial V_{x}}{\partial z}+\frac{\partial V_{z}}{\partial x}
\end{gathered}
$$

and

$$
\frac{\partial \tau_{z z}}{\partial t}=(\lambda+2 \mu) \frac{\partial V_{z}}{\partial z}+\lambda \frac{\partial V_{x}}{\partial x}
$$

where $V_{x}$ and $V_{z}$ are the particle velocities in the $x$ and $z$ directions, respectively, $\tau_{x x}$ and $\tau_{z z}$ are the normal components of stress, and $\tau_{x z}$ is the shear stress. The medium parameters are given by $p$ (density), $\mu$ (rigidity), and $\lambda$ (Lame parameter) and vary as a function of position. Source terms are given by $f_{x}$ and $f_{z}$.

Equations $A 1$ and $A 2$ are temporally staggered by one-half time-step. That is, the numerical solution to Equation $A 1$ is obtained by solving for $V_{x}$ and $V_{z}$ using values of stress computed at the previous half time-step, and these new velocities are used to update $\tau_{x x}, \tau_{z z}$, and $\tau_{x z}$ in the following half-time step. Thus, the elastic wave equation can be broken into two sets of independent first-order differential equations, where the velocities depend only on the stresses and the stresses depend only on the velocities. There are 5 unknown variables in this system. 
Writing the wave equation as two sets of 1 st order equations is advantageous because the medium parameters are no longer functions of the spatial derivatives. This simplifies the solution for inhomogeneous media, and results in lower computer storage requirements. Although the conventional method of writing the 2-D elastic wave equation as a second-order vector system involves only two variables (the displacements in the $x$ and $z$ directions), in inhomogeneous media additional memory is required for computationally efficient solutions to avoid recalculation of terms. In addition, Equations $\mathrm{A} 1$ and $\mathrm{A} 2$ are conducive to a particular spatial discretization known as a staggered grid implementation.

\section{Staggered Grid:}

We use a finite-difference scheme on a spatially staggered grid (Figure A1). The computational variables $V_{x}, V_{z}, \tau_{x x}, \tau_{z z}$, and $\tau_{x z}$ are computed on separate grids, each staggered by $1 / 2$ node spacing ( $\tau_{x x}$ and $\tau_{z}$ are computed on the same grid). Staggered grids are particularly useful in 1st order systems (odd ordered systems in general) because the finite-difference approximations are more accurate than non-staggered schemes [Fornberg, 1990]. Staggered differences approximate the derivative between grid points, which effectively doubles the density of the grid. They are more accurate than non-staggered differences which approximate the derivative on the grid points and have an accuracy consistent with the actual grid density. Thus, in staggered systems the width of the differencing stencil is necessarily less than its non-staggered counterpart. The discretized spatial approximations to the stress derivatives in Equation A1 are valid at the velocities, while the reverse is true for Equation A2. The higher accuracy of the staggered system translates into less computer memory, since a denser grid will be required to achieve the same accuracy in the conventional case. The major drawback of the staggered grid approach is that this method is significantly. more difficult to implement as a computer code.

\section{Finite Differencing:}

We use a finite-differencing scheme that is 4th order accurate in space and 2nd order accurate in time. Equations $\mathrm{A} 1$ and $\mathrm{A} 2$ can be discretized by

$$
\begin{aligned}
& V_{x}^{m, n}(t)=\Delta t\left(D_{t-\Delta, 2}^{n+0}\left[\tau_{x x}\right]+D_{t-\Delta t / 2}^{m+0}\left[\tau_{x z}\right]\right) / \rho^{m, n}+V_{x}^{m, n}(t-\Delta t) \\
& V_{z}^{m, n}(t)=\Delta t\left(D_{i-\Delta / / 2}^{n+1}\left[\tau_{x z}\right]+D_{t-\Delta t / 2}^{m+1}\left[\tau_{z z}\right]\right) / \rho^{m, n}+V_{z}^{m, n}(t-\Delta t) \\
& \tau_{x x}^{m, n}(t+\Delta t / 2)=\Delta t\left(\left(\lambda^{m, n}+2 \mu^{m, m}\right) D_{t}^{m+1}\left[V_{x}\right]+\lambda^{m, n} D_{t}^{m+0,}\left[V_{z}\right]\right)+\tau_{x x}^{m, n}(t-\Delta t / 2) \\
& \tau_{z z}^{m, n}(t+\Delta t / 2)=\Delta t\left(\lambda^{m, n} D_{t}^{n+1}\left[V_{x}\right]+\left(\lambda^{m, n}+2 \mu^{m, n}\right) D_{t}^{m+0,}\left[V_{z}\right]\right)+\tau_{z z}^{m, n}(t-\Delta t / 2) \\
& \tau_{x z}^{m, n}(t+\Delta t / 2)=\Delta t\left(D_{t}^{m+1} \cdot\left[V_{x}\right]+D_{i}^{n+0}\left[V_{z}\right]\right) \mu^{m, n}+\tau_{x z}^{m, n}(t-\Delta t / 2)
\end{aligned}
$$

where $D_{\mathrm{t}}^{\mathrm{n}}$ and $D_{\mathrm{t}}^{\mathrm{m}}$, represent 4th order staggered grid differencing functions given by

$$
D_{i}^{n}[\mathrm{X}]=\left(c_{1}\left(\mathrm{X}_{i}^{\mathrm{m}, \mathrm{n}}-\mathrm{X}_{1}^{\mathrm{m}, \mathrm{n}-1}\right)+c_{2}\left(\mathrm{X}_{i}^{\mathrm{m}, \mathrm{n}+1}-\mathrm{X}_{i}^{\mathrm{m}, \mathrm{n}-2}\right)\right) / \Delta \mathrm{X}
$$

and

$$
D_{t}^{\mathrm{m}} \cdot[\mathrm{X}]=\left(c_{1}\left(\mathrm{X}_{t}^{\mathrm{m}, \mathrm{n}}-\mathrm{X}_{\mathrm{t}}^{\mathrm{m}-1, \mathrm{n}}\right)+c_{2}\left(\mathrm{X}_{\mathrm{t}}^{\mathrm{m}+1, \mathrm{n}}-\mathrm{X}_{\mathrm{t}}^{\mathrm{m}-2, \mathrm{n}}\right)\right) / \Delta \mathrm{z}
$$

where $c_{1}$ and $c_{2}$ are finite-differencing coefficients given by $c_{1}=9 / 8$ and $c_{2}=-1 / 24$. The 
subscript $t$ represents the time-step and the superscripts $m$ and $n$ represent the grid row and column grid number, respectively (Figure A1).

The spatial discretizations $\Delta x$ and $\Delta z$ depend on the frequency of the source and the lowest velocity in the medium. We've found that for typical problems at least 10 grid points per wavelength are necessary to minimize the effects of numerical dispersion [e.g., Chin et al., 1984]. If the grid is too dense, however, the computational resources will be overtaxed. The temporal time-step $\Delta t$ depends on the Courant limit, and is a function of the highest medium velocity and the node spacing [Press et al., 1986]. For 4th order staggered grid approximations, the Courant condition requires that $\Delta t<0.606 \Delta h / \alpha$, where $\Delta h$ is that minimum grid spacing and $\alpha$ is the maximum velocity [Levander, 1988].

\section{Source Term:}

An explosive source with a time function defined by the derivative of a Gaussian pulse (Ricker wavelet) is used to drive the finite-difference simulation (f in Eq. A-1). An explosive source requires that $f_{x}=f_{z}$. The time history of the pulse is is given by

$$
f(t)=-2 \alpha\left(t-t_{0}\right) \exp ^{-\alpha\left(t-t_{0}\right)^{2}}
$$

where $t_{0}$ is the time at the middle of the pulse and the parameter $\alpha$ controls the frequence content of the source.

The source is applied to a point in the two dimensional grid, and is centered about the normal stress components $\tau_{x x}$ and $\tau_{z z}$.(Figure A1). The finite-difference approximation of the source applied at grid point $m, n$ is given by

$$
\begin{aligned}
& V_{x}^{m+0, n+1}=+\operatorname{Af} \Delta t\left(\Delta x^{2} \Delta z\right) / \rho^{m, n} \\
& V_{x}^{m+0, n+0}=-\operatorname{Af} \Delta t /\left(\Delta x^{2} \Delta z\right) / \rho^{m, n} \\
& V_{z}^{m+0, n+0}=+A f \Delta t /\left(\Delta z^{2} \Delta x\right) / \rho^{m, n} \\
& V_{z}^{m-1, n+0}=-A f \Delta t /\left(\Delta z^{2} \Delta x\right) / \rho^{m, n}
\end{aligned}
$$

where $\mathbf{A}$ is the amplitude of the source. A point source in a 2-dimensional model is computationally equivalent to a line source in 3 dimensions [e.g., Aki and Richards, 1980].

\section{Boundary Conditions:}

A stress-free boundary condition is applied to the upper interface, which emulates the ground surface [e.g., Vidale and Clayton, 1986]. This boundary condition allows reflections and mode conversions to occur from the interface. The requirements are that the shear stress $\tau_{x z}$ and the vertical normal component of stress $\tau_{z z}$ equal 0 . This is implemented in the differencing scheme by requiring that $\tau_{x z}$ and $\tau_{z z}$ be odd functions centered about the surface (thus equal to 0 at the interface). This allows nodes immediately below the surface to use artificial grid points immediately above the surface in the differencing.

First-order elastic absorbing boundary conditions are applied to the side and bottom 
grid boundaries [Clayton and Engquist, 1977, 1980; Emerman and Stephen, 1983; Mahrer, 1986]. Differencing equations are given in Clayton and Engquist [1977]. We use paraxial methods to extrapolate the wave field normally outward from each boundary. Interior grid points can utilize these extrapolated values in the differencing scheme. Waves normal to each boundary are "absorbed". completely. Waves hitting a boundary at an angle exhibit partial reflection back into the grid (including mode conversion). Depending on the incidence angle, the amplitude of the reflected waves are usually less than $5-10 \%$ of the incident wave.

\section{Visualization:}

It is useful to graphically represent the wave field computed over the 2-D finitedifference grid. Seismic waves in an elastic medium can be decomposed into the $P$ and $S$ potentials (corresponding to the compressional and shear velocities). The $P$ potential is similar to the pressure field from acoustic wave propagation in water. The $S$ potential represents a shearing of the elastic medium and is similar to the side-byside motion associated with fault slip. The $P$ and $S$ potentials are proportional to the dilatation and shear rotation, respectively. Within a scale factor, the $P$ potential (actually it's time derivative) is obtained from the particle velocities by

$$
P=\frac{\partial V_{x}}{\partial x}+\frac{\partial V_{z}}{\partial z}
$$

and the $\mathrm{S}$ potential (time derivative) is obtained by

$$
S=\frac{1}{2}\left(\frac{\partial V_{x}}{\partial z}-\frac{\partial V_{z}}{\partial x}\right)
$$

P-to-S and S-to-P conversions occur wherever seismic waves engage the free surface or medium boundaries.

It is particularly useful to display the $P$ and $S$ potentials in real time during the finitedifference simulations, and we have developed this capability using an $\mathrm{X}$ Windows interface. This type of display allows mode conversions to be viewed at medium boundaries, and to quickly investigate the cause of numerical instabilities in the simulations. The computational effort for this real-time visualization is not large compared to the velocity/stress updates (the visual updates are made at intervals of several time-steps).

\section{Computer Requirements:}

The numerical simulations used in this study were performed on a Sun Sparc 10-41 workstation ( $40 \mathrm{MHz}$ ) with $64 \mathrm{MBytes}$ of memory and a $1 \mathrm{MByte}$ data cache. The finite-difference grid utilized 375,000 computational nodes, with simulations lasting 3333 time-steps. The CPU time for a single run was approximately 70 minutes. Thus, the average time per node per time-step was $3.4 \mu \mathrm{s}$. However, due to a dynamic grid algorithm (see below) the actual number of calculations were reduced by $30 \%$. Thus, the real computational time per node per time-step was $4.8 \mu \mathrm{s}$. A single computational node requires 64 floating point operations for each time-step. This gives a sustained operating speed of 13.5 MFLOPS (floating point operations per second). Computations were made in single precision. 
Depending on the medium parameters, the memory requirement per node is between 20 and 32 bytes (5-8 single precision words). Approximately 12 MBytes were required for our simulations. The algorithm and computational speed is efficient enough, so that memory is the limiting factor in determining problem size.

The code was vectorized and ported to a Cray YMP at the National Energy Research Supercomputer Center (NERSC). The run-time on this machine was reduced by a factor of about 23 from identical runs on a Sparc 10-41 workstation.

The computational efficiency was increased using 3 techniques: (1) Dynamic grid algorithm; (2) Vectorization; (3) Compiler optimization. A dynamic grid algorithm was used to reduce the total number of computations. Because the wavefront covers only a small region of the grid during the early phase of the simulation, a considerable speedup was obtained by eliminating from the computation the region of the grid yet to be activated by the seismic pulse (zero elements are not computed). In general this improvement is problem dependent, but we found speedups of 1.4 to 2.0 to be typical. Vectorization of the finite-difference code improved the performance, even on scalar machines. Although the Sun Sparc 10-41 workstation does not contain a vector processor, a speedup of about 1.7 was observed using a vectorized version of the algorithm. This improvement is attributed to local memory referencing [e.g., Dowd, 1993]. When an algorithm is designed so that a higher percentage of variables are found in the data cache or CPU registers, memory access time is significantly reduced. Finally, we found that using different optimizing compilers had a significant effect on performance. For example, a speedup of 1.8 was achieved when the lowest and most computationally intensive section of the code was programmed in FORTRAN rather than the $C$ language. Overall, we were able to increase the algorithms efficiency by a factor of about 5 by simply appling proper programming strategies. 


\section{REFERENCES}

Aki, K., and P. Richards, Quantitative Seismology, W. H. Freeman and Company, New York, 932 pp., 1980.

Charrette, E. E., 3D acoustic finite difference on a nCUBE massively parallel computer, nCUBE Technical Report 116, 12 pp., 1992.

Chin, R. C. Y., G. Hedstrom, and L. Thigpen, Numerical methods in seismology, J. Comput. Phys., 54, 18-54, 1984.

Claerbout, J., Imaging the Earth's Interior, Blackwell Scientific Publications, 398 pp., 1985.

Clayton, R., and B. Engquist, Absorbing boundary conditions for acoustic and elastic wave equations, Bull. Seismol. Soc. Am., 67, 1529-1540, 1977.

Clayton, R., and B. Engquist, Absorbing boundary conditions for wave-equation migration, Geophysics, 45, 895-904, 1980.

Dowd, K.; High Performance Computing, O'Reilly and Associates, Inc., 371 pp., 1993.

Emerman, S. H., and R. A. Stephen, Comment on "Absorbing boundary conditions for acoustic and elastic wave equations," by R. Clayton and B. Engquist, Bull. Seismol. Soc. Am., 73, 661-665; 1983.

Fornberg, B, High-order finite differences and the pseudospectral method on staggered grids, SIAM J. Numer. Anal., 27, 904-918, 1990.

Frankel, A., and J. Vidale, A 3-dimensional simulation of seismic waves in the Santa Clara Valley, California, from a Loma-Prieta aftershock, Bull. Seismol. Soc. Am., 82, 2045-2074, 1992.

Graves, R. W., R. W. Clayton, Modeling path effects in three-dimensional basin structures, Bull. Seismol. Soc. Am., 82, 81-103, 1992.

Hedstrom, G., The selection of mesh size for long-range acoustics computations, Lawrence Livermore National Laboratory UCRL, submitted, 1993.

Kelly, K. R., R. W. Ward, S. Treitel, and R. M. Alford, Synthetic seismograms: A finite-difference approach, Geophysics, 41, 2-27, 1976.

Kneib, G., and C. Kerner, Accurate and efficient seismic modeling in random media, Geophysics, 58, 576-588, 1993.

Levander, A. R., Fourth-order finite-difference P-SV seismograms, Geophysics, 53, 1425-1436, 1988.

Madariaga, R., Dynamics of an expanding circular fault, Bull. Seismol. Soc. Am., 66, 639-666, 1976.

Mahrer, K. D., An empirical study of instability and improvement of absorbing 
boundary conditions for the elastic wave equation, Geophysics, 51, 1499-1501, 1986.

Press, W. H., B. P. Flannery, S. A. Teukolsky, and W. T. Vetterling, Numerical Recipes, Cambridge University Press, Cambridge, 818 pp., 1986.

Vidale, J. E., and R. W. Clayton, A stable free-surface boundary condition for twodimensional elastic finite-difference wave simulation, Geophysics, 51, 2247-2249, 1986.

Virieux, J., P-SV wave propagation in heterogeneous media: Velocity-stress finitedifference method, Geophysics, 51, 889-901, 1986. 


\section{FIGURE CAPTIONS}

Figure 1: Crustal model. The model is a 2-D cross section extending $6 \mathrm{~km}$ in the horizontal direction and $1 \mathrm{~km}$ in depth. The $P$-wave velocities are $5 \mathrm{~km} / \mathrm{sec}$ at the top and $7 \mathrm{~km} / \mathrm{sec}$ at $1 \mathrm{~km}$ depth (a uniform gradient of $2 \mathrm{~km} / \mathrm{sec}$ ). The stippled region in the center is the low-velocity rubble zone (P-wave velocity $2.0 \mathrm{~km} / \mathrm{sec}$ ). Along the surface are seismometers separated every $0.2 \mathrm{~km}$ (triangles). The source is a compressional Richer wavelet (star). The curves represent the high-frequency raypaths from the source to each receiver.

Figure 2: Expanding wave fields of the $P$ (red) and $S$ (green) potentials as a function of time. The 5 images show the wave field at 0.15 (top), $0.3,0.45$, and 0.6 (bottom) seconds. Blue tint indicates negative amplitude. Various phases are observed including the direct $\mathrm{P}$ arrival (P), the P-to-S conversion along the free-surface (PS), and the Rayleigh wave (R). Due to the shallow depth of the source, a direct shear wave (S) is created by the interaction of the source with the free-surface. The delayed (Pd) and refiected ( $\mathrm{Pr}$ ) $\mathrm{P}$ arrivals are clearly observed as the wave field interacts with the lowvelocity rubble zone. Non-absorbed (artificial) reflections off the bottom and side grid boundaries are noticeable during the 0.45 and 0.6 second wave fields.

Figure 3: Synthetic seismograms along the surface ( $P$ potential) for the low-velocity zone model. The clipped portion corresponds to the surface wave.

Figure 4: Differential synthetic seismograms along the surface ( $P$ potential). Shown are the difference in synthetics between simulations with and without the low-velocity zone. The amplitude is the same as Figure 3 . This plot indicates the effect of the low-velocity zone.

Figure 5: Synthetic seismograms along the surface (P potential) for the low-velocity zone model with $5 \%$ noise added to the velocity structure.

Figure 6: Synthetic seismograms along the surface (P potential) for the low-velocity zone model with $10 \%$ noise added to the velocity structure.

Figure 7: Seismic wave fields as a function of time for the low-velocity zone model with $10 \%$ noise added to the velocity structure (see Figure 2). Note the perturbations as the wave fields begin to lose their coherency. The seismic delay and the reflection from the void are clearly observed, although the effect is not as defined as in the unperturbed model shown in Figure 2.

Figure 8: Synthetic seismograms along the surface ( $P$ potential) for the low-velocity zone model with $20 \%$ noise added to the velocity structure.

Figure A1: Staggered grid implementation of finite-difference scheme. The $x$ axis is horizontal and the $z$ axis is vertical. The separation between nodes in the $x$ and $z$ directions, are $\Delta x$ and $\Delta z$, respectively $(\Delta x=\Delta z)$. The variables $V_{x}, V_{z}, \tau_{x x}, \tau_{z x}$, and $\tau_{x z}$ are calculated on separate grids, each staggered by $1 / 2$ node spacing $\left(\tau_{\mathbf{x x}}\right.$ and $\tau_{z z}$ are calculated on the same grid). The row number is given by $m$, while the column number is given by $\mathbf{n}$. 


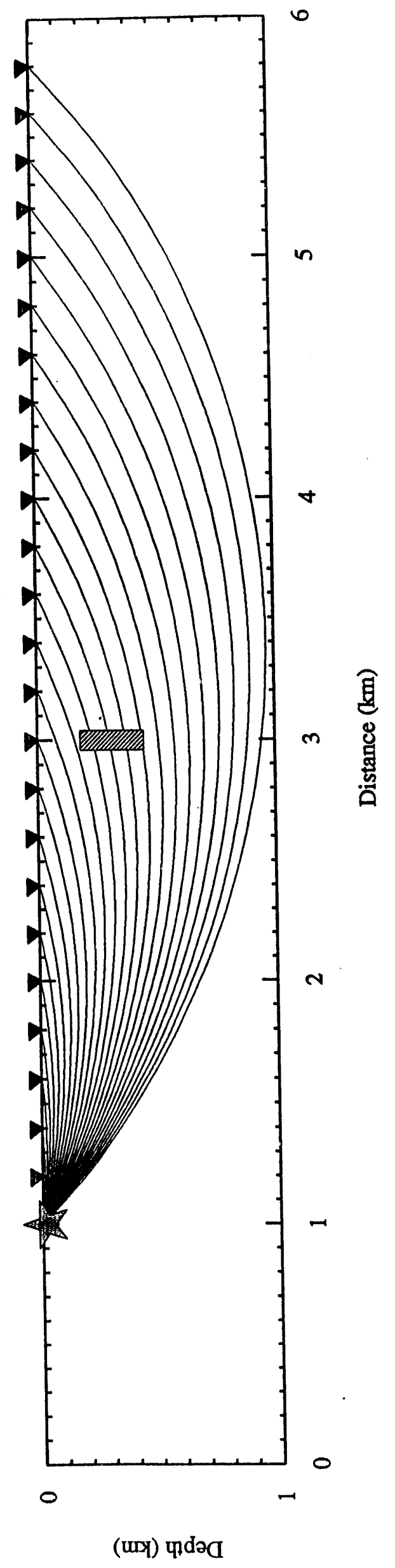

Figure 1 


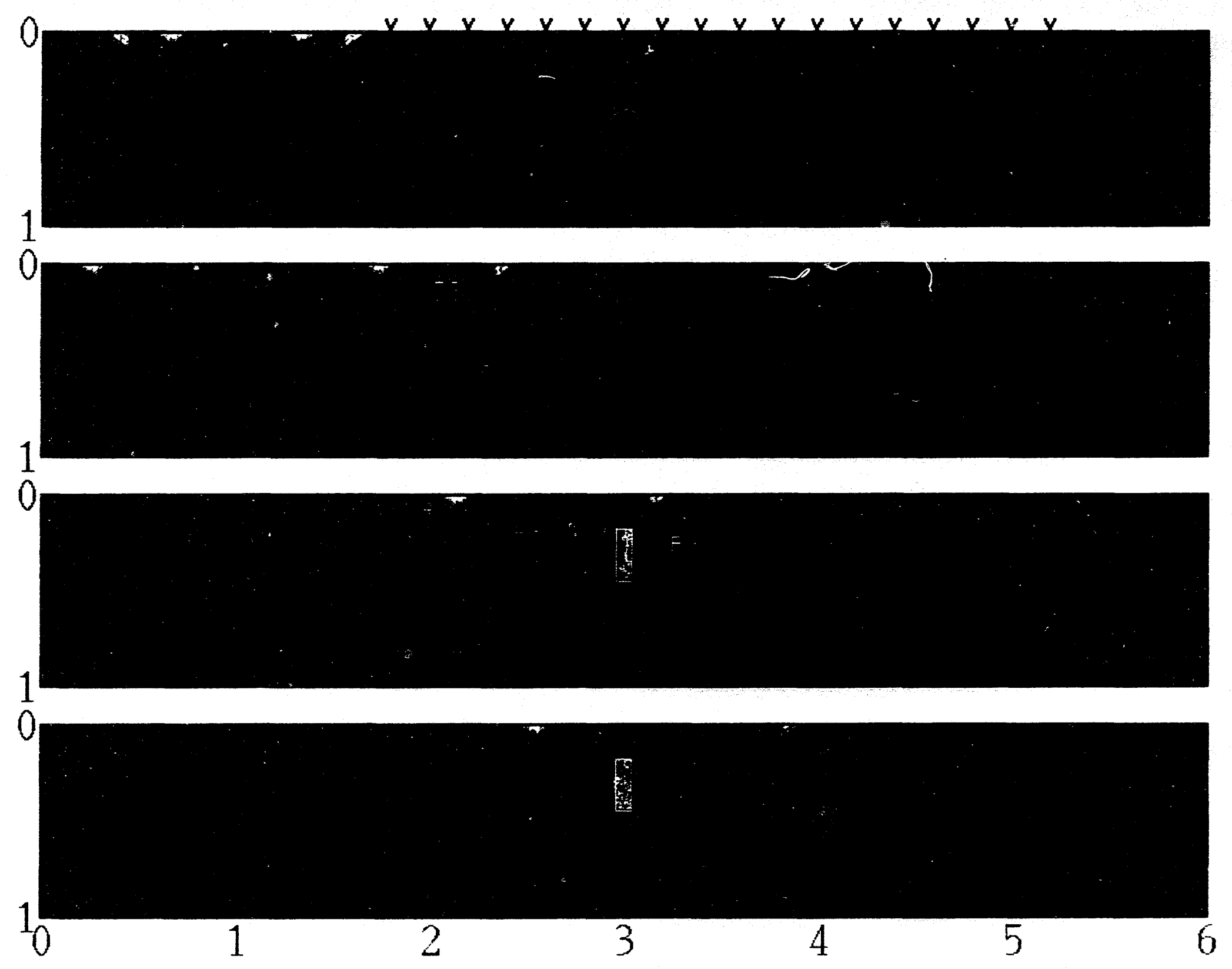

Figure 2 
Propagation Through Rubble Zone

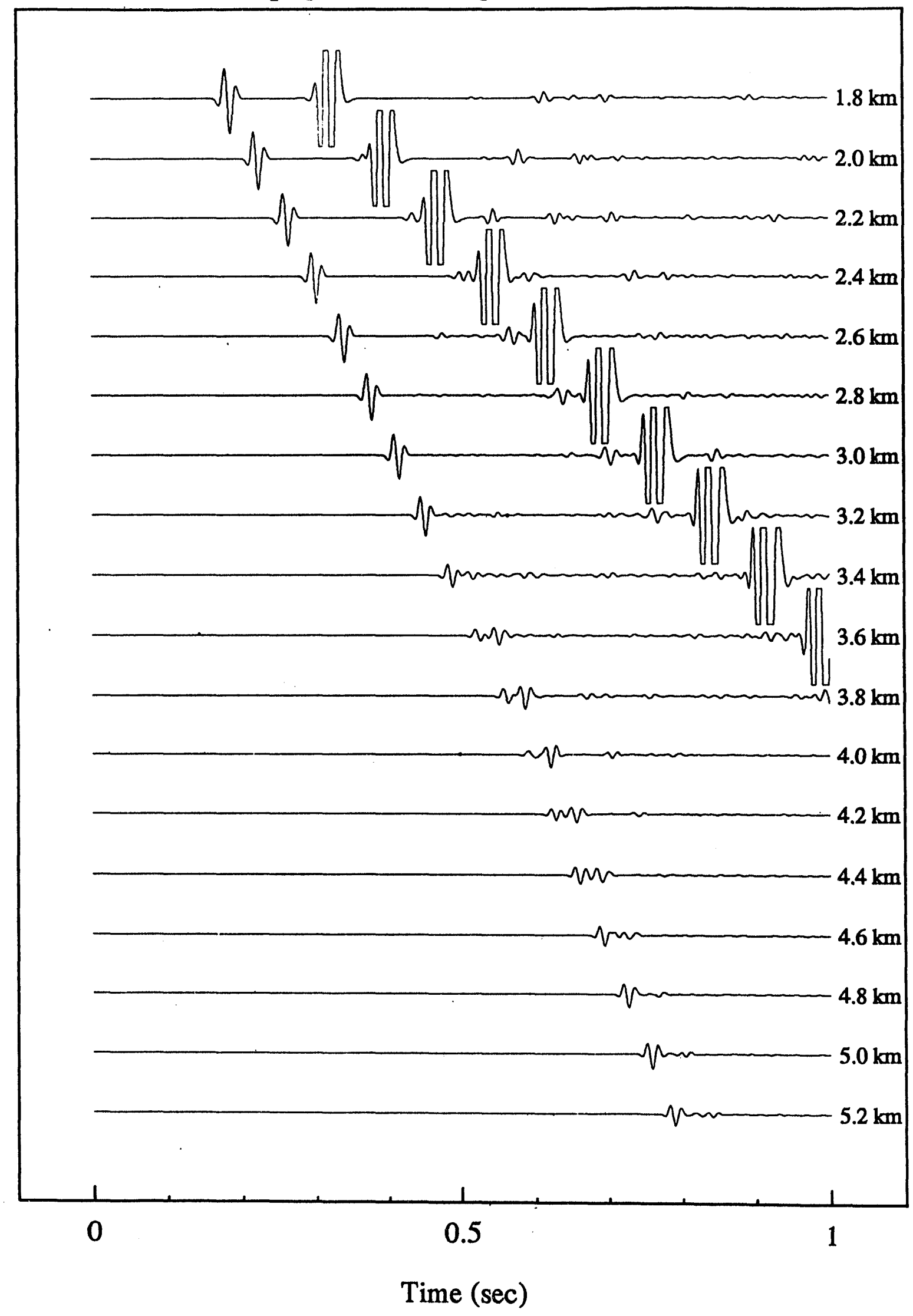

Figure 3 
Differenced Propagation Through Rubble Zone

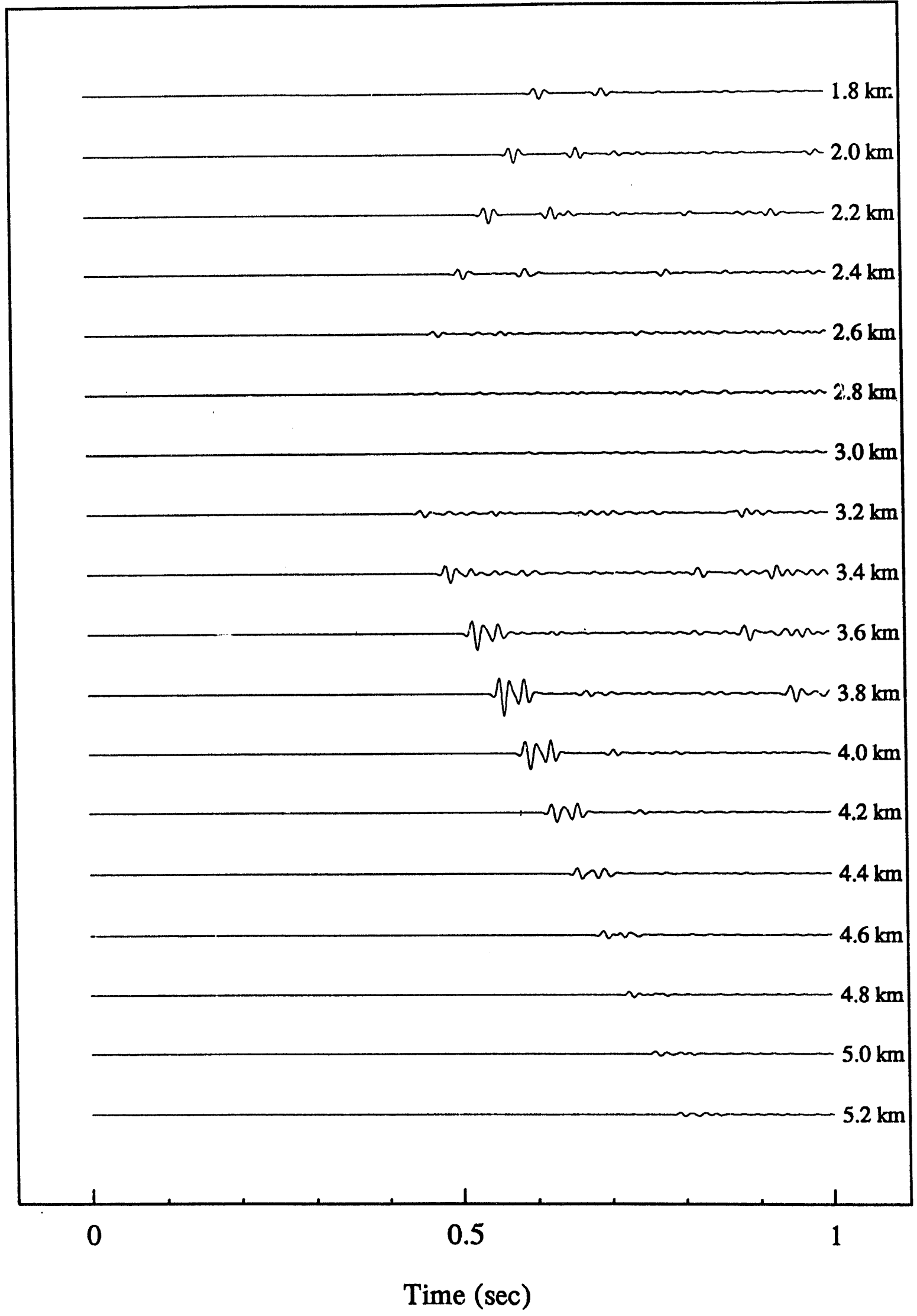

Figure 4 


\section{Propagation Through Rubble Zone With 5\% Noise}
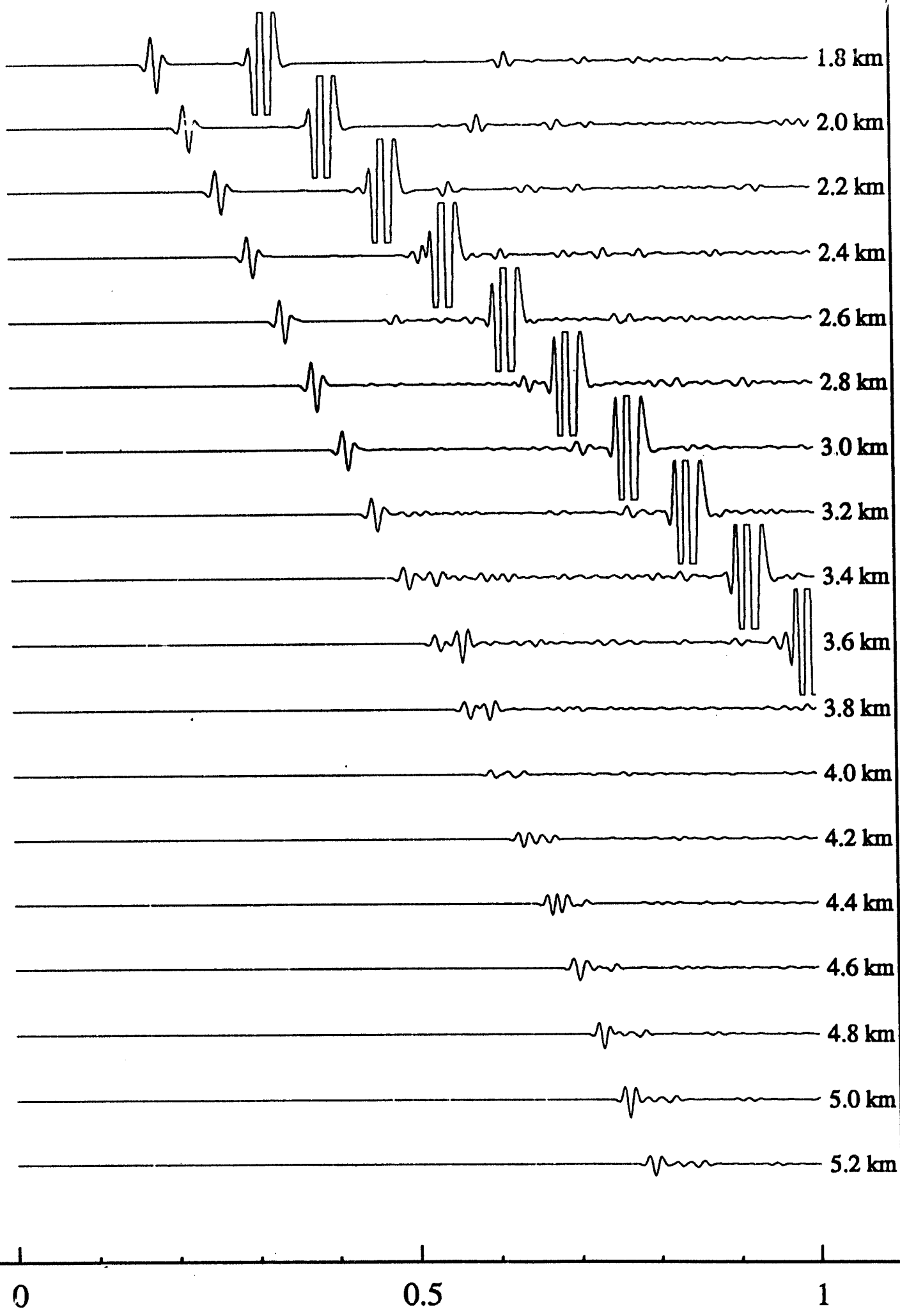

Time (sec)

Figure 5 
Propagation Through Rubble Zone With 10\% Noise

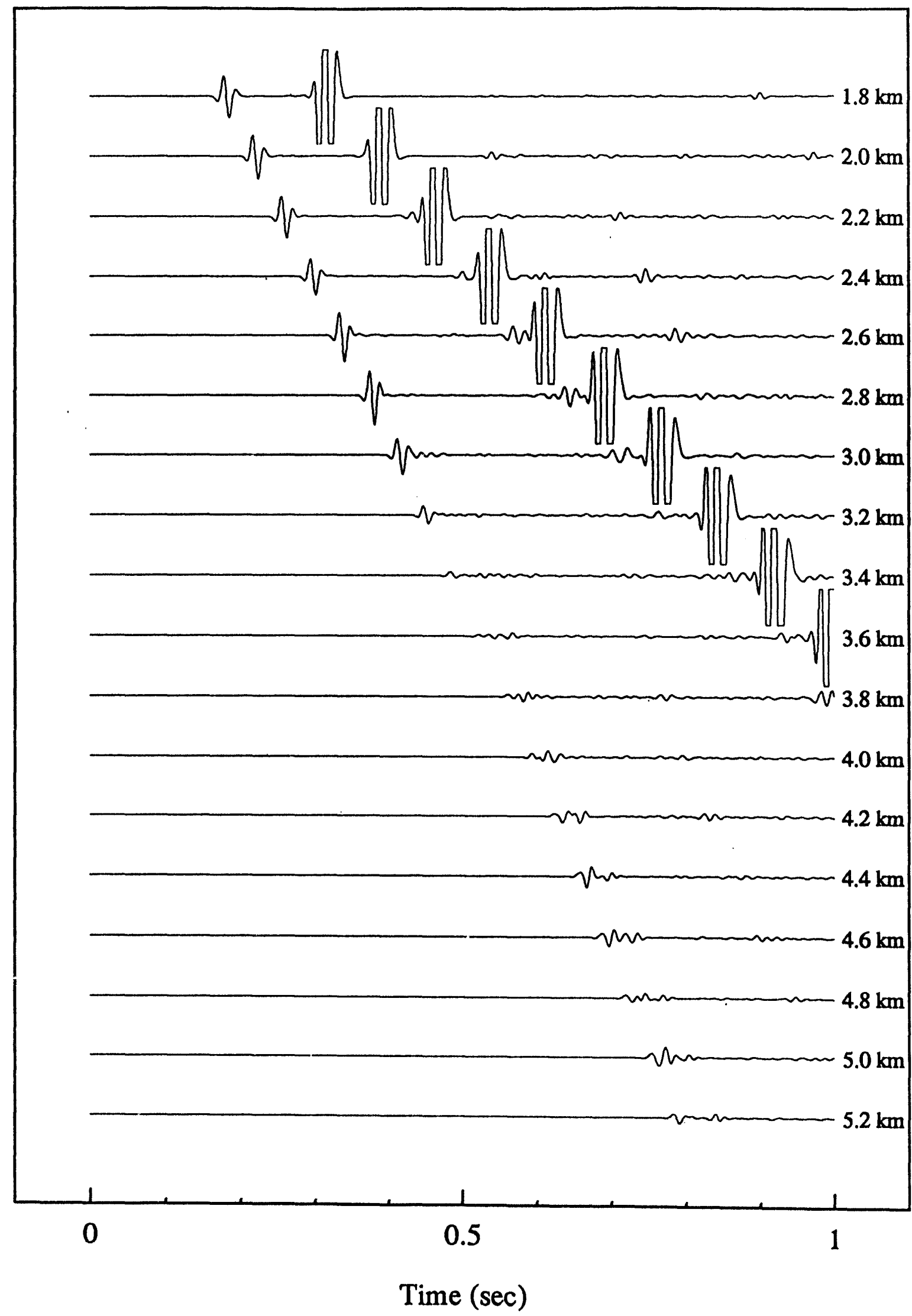

Figure 6 


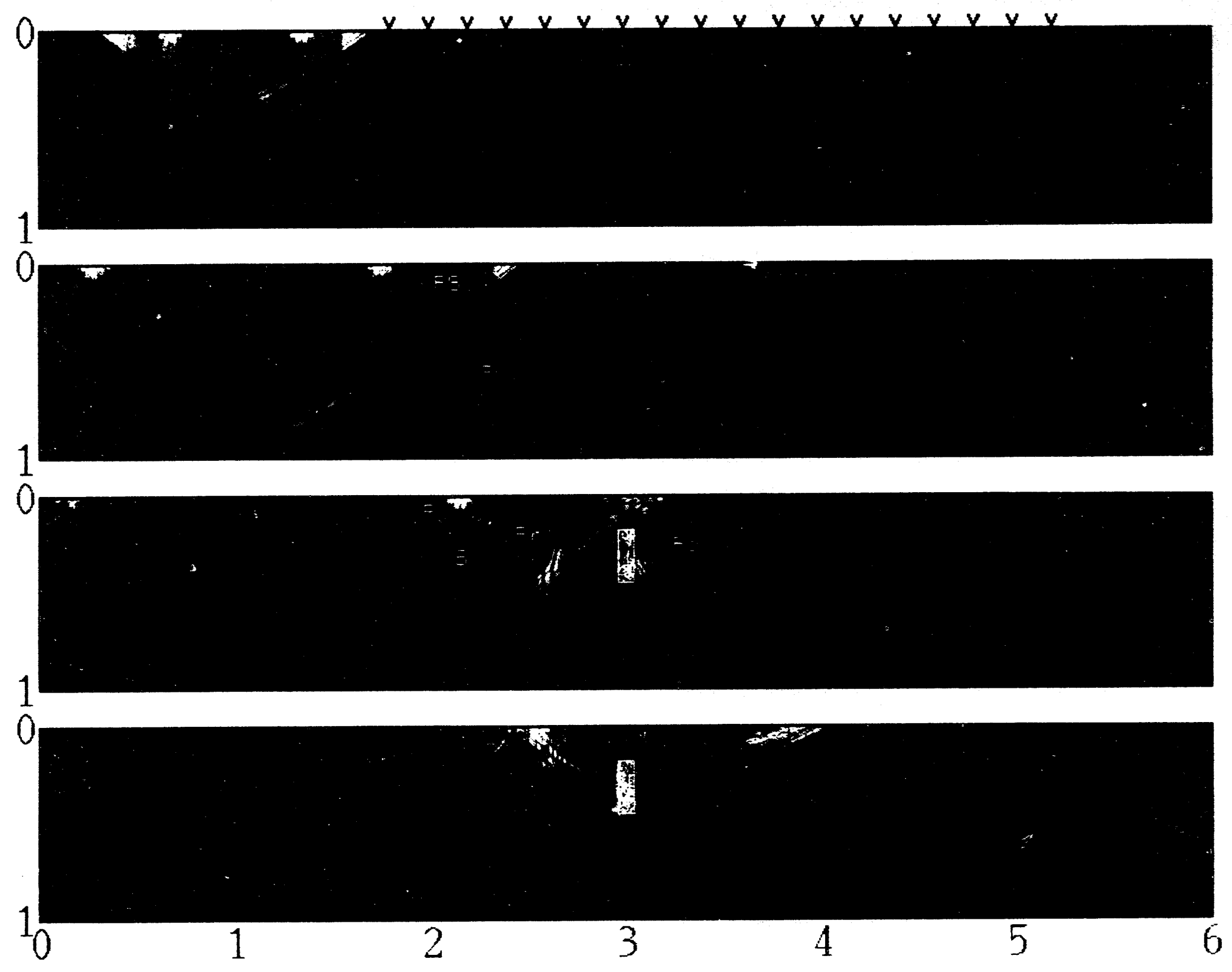

Figure 7 


\section{Propagation Through Rubble Zone With 20\% Noise}

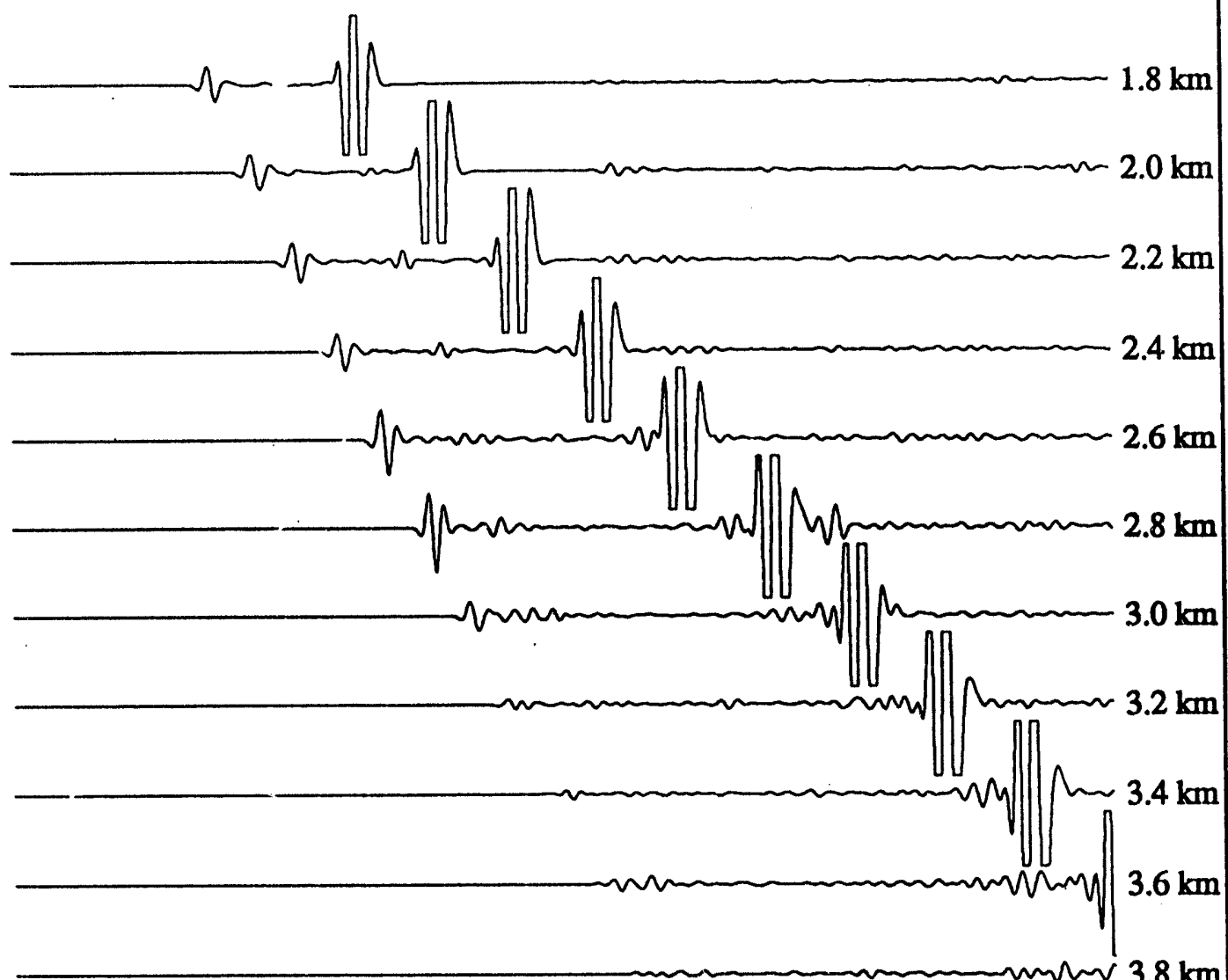

$3.8 \mathrm{~km}$

$\sim 4.0 \mathrm{~km}$

$4.2 \mathrm{~km}$

$4.4 \mathrm{~km}$

$4.6 \mathrm{~km}$

$4.8 \mathrm{~km}$

$5.0 \mathrm{~km}$

$5.2 \mathrm{~km}$

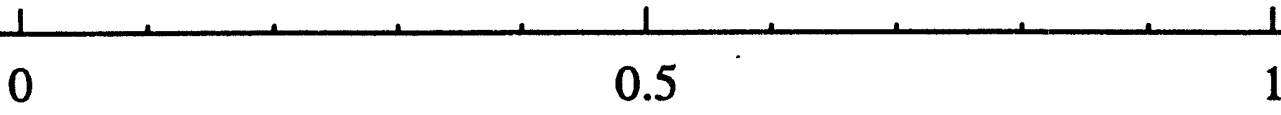

1

Time (sec)

Figure 8 

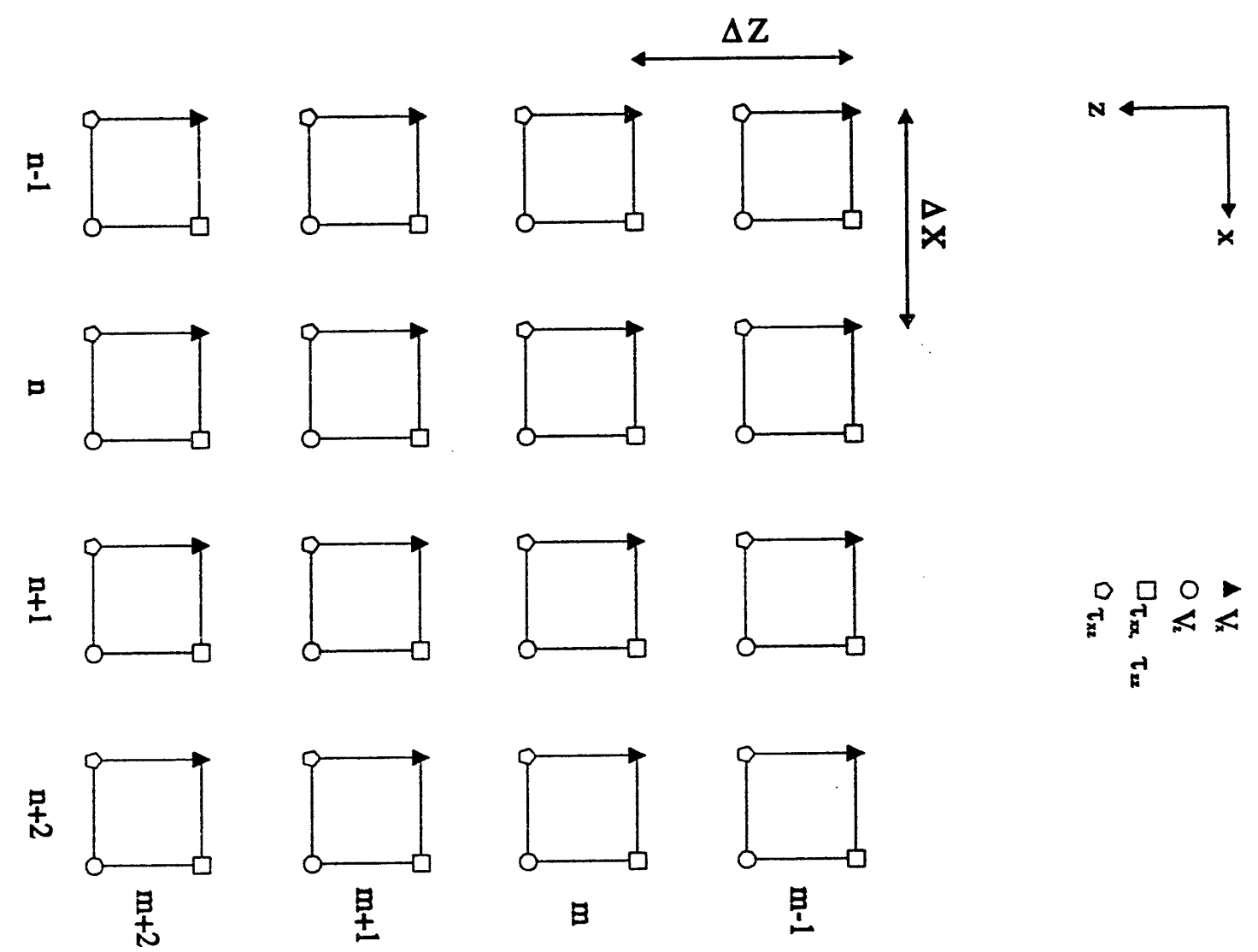

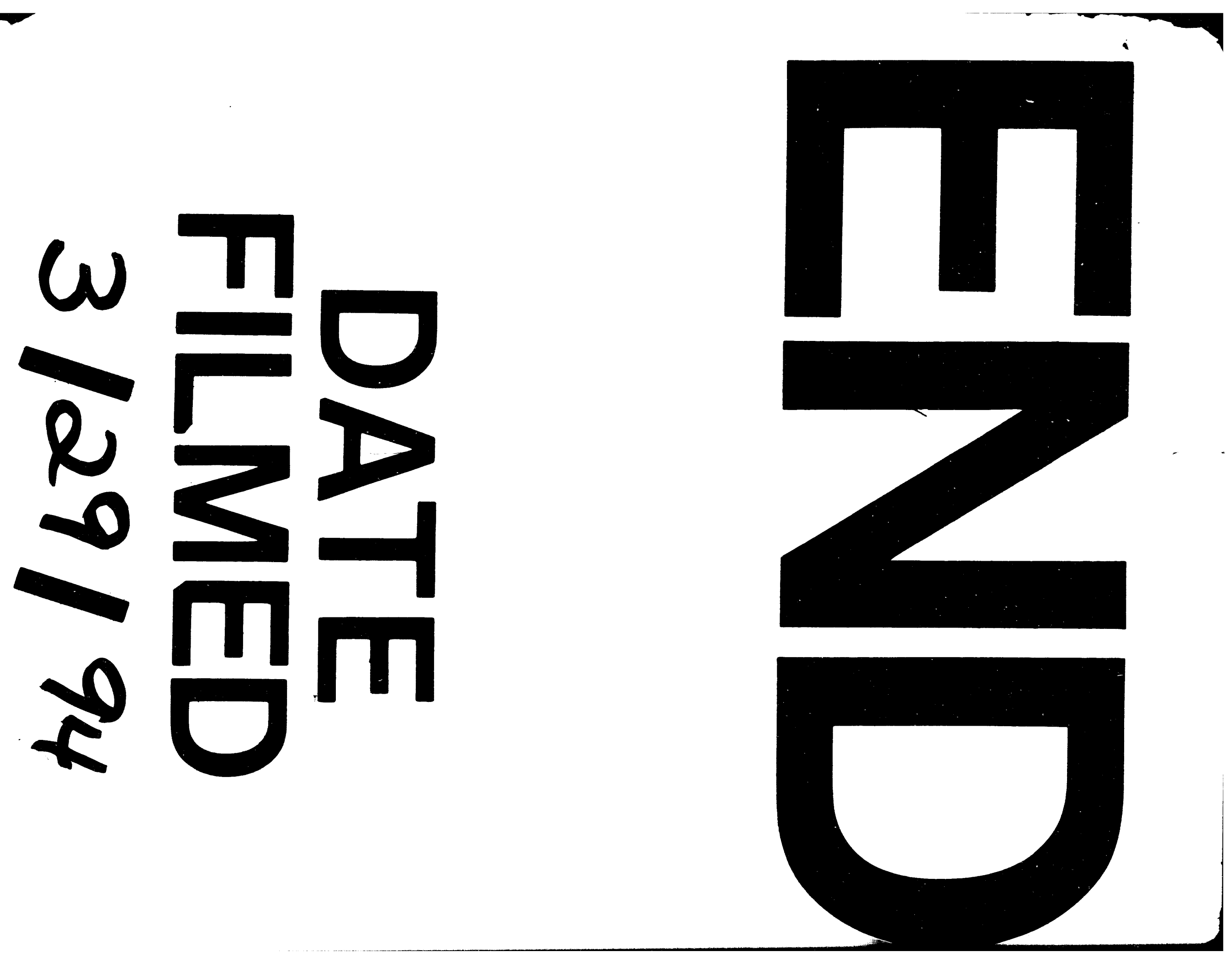
